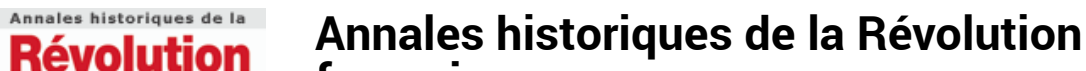 \\ française française
}

332 | avril-juin 2003

Une révolution du pouvoir exécutif?

\section{Rüdiger HILLMER, Die napoleonische Theaterpolitik.} Geschâftstheater in Paris 1799-1815, Köln, Bôhlau, 1999, $536 \mathrm{p}$.

Thomas Höpel

\section{(2) OpenEdition \\ Journals}

Édition électronique

URL : https://journals.openedition.org/ahrf/5713

DOI : $10.4000 / a h r f .5713$

ISSN : 1952-403X

Éditeur :

Armand Colin, Société des études robespierristes

Édition imprimée

Date de publication : 1 juin 2003

Pagination : 217-219

ISSN : 0003-4436

Référence électronique

Thomas Höpel, « Rüdiger HILLMER, Die napoleonische Theaterpolitik. Geschâftstheater in Paris

1799-1815, Köln, Bôhlau, 1999, 536 p. », Annales historiques de la Révolution française [En ligne], 332 |

avril-juin 2003, mis en ligne le 22 avril 2008, consulté le 24 avril 2022. URL : http://

journals.openedition.org/ahrf/5713; DOI : https://doi.org/10.4000/ahrf.5713

Ce document a été généré automatiquement le 24 avril 2022.

Tous droits réservés 


\title{
Rüdiger HILLMER, Die napoleonische Theaterpolitik. Geschâftstheater in Paris 1799-1815, Köln, Bôhlau, 1999, $536 \mathrm{p}$.
}

\author{
Thomas Höpel
}

La nouvelle politique culturelle et artistique qui fut instaurée pendant la Révolution de 1789 a connu dans les années qui suivirent le bicentenaire un regain d'intérêt dans la recherche. En témoignent les différentes monographies et volumes collectifs consacrés en particulier au marché du livre et à la presse (Caria HESSE, Publishing and Cultural Politics in Revolutionary Paris, 1789-1810, Berkeley - Los Angeles - Oxford, 1991) ainsi qu'à la création de musées (Ingeborg CLEVE, Geschmack, Kunst und Konsum. Kulturpolitik als Wirtschaftspolitik in Frankreich und Wiirttemberg 1805-1845, Gôttingen, 1996 ; Gottfried FLIEDL (Hrsg.), Die Erfindung des Museums. Anfânge der burgerlichen Museumsidee in der franzosischen Revolution, Wien (Museum zum Quadrat), 1996 ; Dominique POULOT, Musée, nation, patrimoine, 1789-1815, Paris, 1997 ; Andrew Mac Clellan, Inventing the Louvre: art, politics and the origins of the modern museum in eigtheenth-century Paris, Cambridge, 1999). Dans ces travaux, les années d'après 1799, qui avaient été souvent traitées en parent pauvre jusque-là, font l'objet d'un véritable examen. L'ouvrage d'Hillmer sur la politique napoléonienne concernant le théâtre, version revue de sa thèse de doctorat de 1997, s'insère dans cette conjoncture de recherches et ouvre en même temps un nouveau domaine à peine exploré jusque-là. Hillmer veut montrer que le système théâtral français, tel qu'on le trouvait aux XIX et XX ${ }^{e}$ siècles, c'est-à-dire la coexistence de théâtres subventionnés et de théâtres commerciaux, ne fut pas le fruit du hasard, mais se présente comme un ensemble ordonné, créé en toute connaissance de cause. À son avis, c'est la législation napoléonienne sur le théâtre du début du XIX ${ }^{\mathrm{e}}$ siècle qui marquerait une césure déterminante dans le développement institutionnel du théâtre. Hillmer appuie son argumentation sur l'exploitation d'un grand nombre de sources. Il a dépouillé systématiquement tous les documents du Bureau des Théâtres du ministère de l'Intérieur. À côté de cela, il a examiné les débats du Conseil des Anciens et du Conseil 
des Cinq-Cents concernant la question du théâtre avant 1799, des mémoires et des pamphlets sur le théâtre jusqu'en 1807 ainsi que des critiques dramatiques, des almanachs et quelques hebdomadaires. Son travail se divise en deux parties : dans la première, il traite du système théatral parisien avant 1807 ; dans la seconde, il examine la politique napoléonienne sur le théâtre à partir de 1807. Un appendice présentant des documents importants clôt l'ouvrage.

2 La première partie de ce travail, qui examine le système qui régit le théâtre avant 1807, ne se limite pas aux années qui vont de 1799 à 1807, comme le titre du livre pourrait le laisser supposer, mais est consacrée en grande partie à la politique concernant le théâtre pendant la Révolution. Hillmer montre comment on en vint, après la libéralisation du théâtre en 1791, à transformer le système régissant le théâtre en France, particulièrement à Paris. Toutefois, pour différentes raisons, on essaya très tôt de revenir sur ces mesures de libéralisation. On créa un grand nombre de nouveaux théâtres commerciaux, mais beaucoup, en partie pour des raisons financières, eurent une existence éphémère. Toutefois, ils attirèrent en même temps d'assez larges couches de la population et concurrencèrent les grands théâtres parisiens de haut niveau en offrant une distraction populaire. Quoi qu'il en soit, cette libéralisation du théâtre ne contribua ni à cette divulgation des Lumières et de la culture espérée par les révolutionnaires ni à une stabilisation économique du marché du théâtre. Comme beaucoup de révolutionnaires considéraient le théâtre en premier lieu comme un moyen d'éducation, mais qu'on lui imputait en même temps une subversivité potentielle, cette évolution ne pouvait pas être acceptée sans conteste. Le résultat en fut un retour larvé de la censure qui, par la suite, sous le Consulat, prit forme de lois. Sous le Directoire, d'intenses discussions eurent lieu pour savoir s'il fallait changer le système théâtral en vigueur, parce qu'on considérait les conséquences de la liberté accordée au théâtre comme catastrophiques aussi bien sur le plan moral qu'esthétique. On ne put pas parvenir à légiférer sur ce problème, car, en s'opposant mutuellement, les différents groupes politiques bloquèrent l'élaboration de toute nouvelle loi.

3 Dans la deuxième partie, Hillmer traite des lignes directrices de la politique napoléonienne concernant le théâtre et de leurs conséquences sur les théâtres commerciaux. On voit clairement que cette politique sur le théâtre s'inscrit dans la continuité des discussions ouvertes en 1792. Un régime politique maintenant stabilisé pouvait se permettre de réaliser ce qui avait été demandé en vain auparavant. Le début fut marqué par la réintroduction légale d'une précensure en avril 1800. Hillmer décrit en détail la genèse des décrets de 1807 qui fondaient le système des deux sortes de théâtres et posaient clairement les principes directeurs de la politique napoléonienne en ce domaine. En réduisant le nombre des théâtres et en limitant la variété de leur répertoire, on voulait parvenir à deux fins : d'abord permettre aux grands théatres et aux quelques théâtres commerciaux d'avoir un budget en équilibre, ensuite renforcer la fonction culturelle des grands théâtres parisiens qui servaient à afficher la supériorité culturelle de la France. Même si la protection d'une culture de haut niveau avait la priorité dans la politique napoléonienne sur le théâtre, ces lois n'en faussèrent pas moins les rapports de l'État avec les théâtres. Des éléments du système de concurrence établi au début de la Révolution furent conservés, même si le triomphe du théâtre commercial parisien fut freiné par la politique napoléonienne concernant le théâtre. La genèse des décrets de 1807 montre aussi clairement que Napoléon ne s'appuyait pas sur une seule instance pour élaborer ses lois. En effet, le texte définitif 
de la loi du 8 août 1807 écartait des propositions préparées auparavant au ministère de l'Intérieur dont dépendait pourtant le théâtre.

4 La politique napoléonienne concernant le théâtre, telle que la représente Hillmer, correspond en grande partie à celle qui fut également suivie dans les autres domaines culturels. On pourrait ici, à titre d'exemple, citer la politique pratiquée envers la presse : avec le décret de 1810 concernant le marché du livre, on réduisit le nombre des imprimeurs et les nouvelles parutions furent soumises à des dispositions légales afin de stabiliser financièrement cette branche secouée par la crise. À côté de cela, le décret introduisit une aggravation des mesures de surveillance.

Il est clair que la politique culturelle de Napoléon en ce qui concerne la réorganisation institutionnelle et légale du paysage culturel et artistique se situe dans la continuité des discussions commencées en 1792. Aussi aurait-il été souhaitable que Hillmer, à certains moments, compare les résultats obtenus dans le domaine du théâtre avec les évolutions constatées dans la politique culturelle en général. De même, sa remarque répétée, selon laquelle la France aurait été le premier pays à introduire les droits d'auteur en 1791, aurait pu être nuancée si Hillmer s'était référé au travail de Caria Hesse sur cette question. Car les lois sur les droits d'auteur de 1791 et de 1793 n'avaient accordé, en fin de compte, qu'un droit de propriété limité aux auteurs, mais renforcé ceux du public et de la nation pour tout ce qui touchait à l'héritage des idées et à la production intellectuelle de l'époque. C'est seulement la loi de 1810 qui introduisit de nouveau la privatisation de l'identité légale de l'auteur. D'autre part, il existait depuis 1709 en Angleterre des dispositions légales sur les droits d'auteur (Mark ROSE, «The Autor as Proprietor: Donaldson v. Beckett and the Genealogy of Modern Authorship ", dans Representations 23 [1988]).

Hillmer a fait un bon travail en ce qui concerne la genèse du système théâtral parisien comme machinerie de divertissement à partir de 1791, et dans laquelle les théâtres commerciaux jouent un rôle important. Il éclaire les nouvelles structures d'entreprise, les programmes offerts ainsi que les nouvelles stratégies de mise en scène. Même si cette «nouvelle culture théatrale de masse » (p.361) fut l'objet de violentes attaques de la part des critiques et du public cultivé, il y avait cependant déjà très tôt des partisans d'une offre correspondant à la demande. Pourtant, c'est justement à l'époque napoléonienne que la plupart des acteurs et des nouveaux théâtres commencèrent à jeter un regard sans illusion sur le niveau culturel de la production dramatique postrévolutionnaire. De même, on constate, dans le domaine du théâtre depuis 1792, une centralisation croissante qui fut encore accentuée par le régime napoléonien. Cette constatation recoupe les connaissances que nous avons de la politique pratiquée dans les autres domaines culturels.

7 En définitive, il s'agit d'une étude établie sur des documents d'archives; elle défriche un terrain négligé jusque-là et confirme les résultats obtenus dans d'autres domaines. Hillmer concentre son attention surtout sur la politique de l'État vis-à-vis des théâtres, mais l'influence des représentations théâtrales sur le public est à peine effleurée. La constatation de Hillmer selon laquelle le théâtre commercial de l'époque napoléonienne, éloigné de toute propagande ou opposition, aurait été complètement apolitique, s'appuie ici sur des travaux précédents qui datent, dans leur ensemble, de la fin du XIX ${ }^{e}$ siècle et dont les conclusions mériteraient d'être remises en question par de nouvelles investigations. 